\title{
Research on Hamilton Graph Determining Algorithm
}

\section{LIU Hai ${ }^{1, a}$,TIAN Ying $\mathrm{Ai}^{2, \mathrm{~b}}$ HU Fang Feng ${ }^{3, \mathrm{c}}$,Jin Jiong Xing ${ }^{4, \mathrm{~d}}$}

\author{
${ }^{1,2,4}$ School of Computer Science, Beijing Information Science and Technology University, Beijing, \\ China, 100101
}

${ }^{3}$ School of Economics and Management, Beijing Information Science and Technology University, Beijing, China, 100192

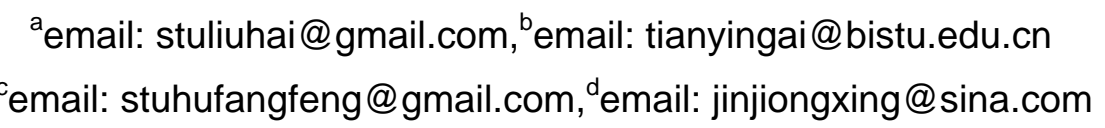

Keywords: Hamilton graph, Heuristic Search algorithm

\begin{abstract}
This Paper sums up the necessary conditions to discriminate undirected Hamilton graph based on the in-depth research into the graph. The heuristic search strategy corresponding to the new necessary conditions is designed to quickly solve the problem of "determination of undirected Hamilton graph". The experimental results show that the heuristic search algorithm can effectively improve computing speed and make accurate determination of "undirected Hamilton graph".
\end{abstract}

\section{Introduction}

As the theoretical research and practical application of the graph theory are continuously deepening and expanding, the graph theory is widely used in operations research, information theory, network theory, biology, chemistry, physics, social sciences, linguistics, computer science and other fields, and is successful in solving some practical problems ${ }^{[1]}$.

Hamilton graph is a special kind of connected graph. In 1895, Irish mathematician Hamilton first proposed the "global travel" problem. He used 20 vertices of a dodecahedron to represent the world's 20 major cities, and the travelers were required to go along the edges of the dodecahedron, starting from one of the cities and passing by all other cities (each city only once), and finally return to the starting point. This is the famous Hamilton graph problem ${ }^{[2]}$.

Hamilton graph's judgment is very difficult, it has proven to be a NP (nondeterministic polynomial) problem. Currently, there are a lot of relevant researches ${ }^{[3-13]}$. Through ingenious application of Hamilton graph's characteristics, the Paper presents an efficient heuristic determination algorithm, which helps make a quick judgment for the specified undirected graph.

This Paper is organized as follow: firstly some relevant preliminary knowledge is introduced in the second section. In the third section, a new necessary condition for determination of the Hamilton graph is given, followed by a detailed description of the heuristic algorithm made according to the condition in the fourth part. The fifth section describes the experimental results, and finally a summarization is made in the sixth section.

\section{Preliminary Knowledge}

Definition 1. The cycle passing by each vertex of the undirected graph $G$ once and only once is called Hamilton cycle.

Definition 2. If the undirected graph $G$ includes at least one Hamilton cycle, the graph $G$ is called Hamilton graph.

A Hamilton graph $G$ includes at least one Hamilton cycle, if the graph $G$ contains only one Hamilton cycle, it is denoted as graph $H$. Generally speaking, to determine a Hamilton graph, you only need to find a Hamilton cycle in a given undirected graph $G$ or if there is no one to be found, it is not a Hamilton graph ${ }^{[14]}$. Following are two simple examples. Figure 1 is a graph $H$ containing 
six vertices. Similarly, Figure 2 is a more general Hamilton graph containing six vertices and more than one Hamilton cycle.

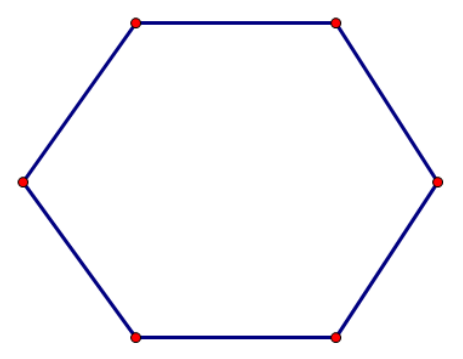

Fig. 1 Graph $H$ containing six vertices

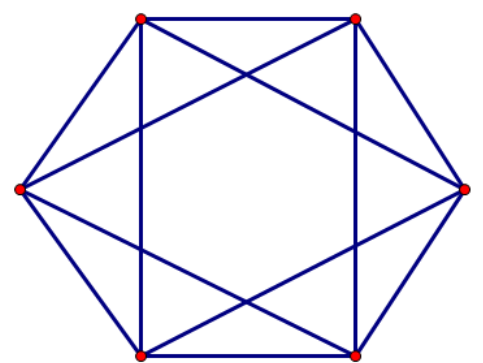

Fig. 2 Hamilton graph containing six vertices

\section{A New Necessary Condition}

Definition 3. The point set $V_{i}^{2 n}$ in the graph $G$ has the following characteristics:

1. The vertices of the point set are all adjacent to vertex $v_{i}$

2. The vertices' degree of point set are all greater than or equal to $n$

If the graph $G$ is a Hamilton graph, the point set $V_{i}^{23}$ is not empty and $d_{i} \geq 3$, then the vertex $v_{i}$ is marked as an active vertex. Here is a simple example. Figure 3 is an undirected Hamilton graph containing six vertices and the active vertices are marked with red in the figure.

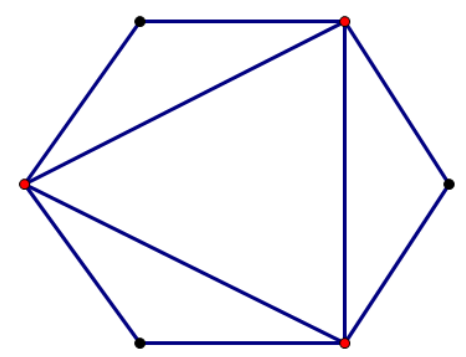

Fig. 3 An undirected Hamilton graph containing six vertices, with the active vertex shown in red Theorem 1. If the graph $G$ is a Hamilton graph, and the active vertex $v_{i}$ meets $\left|V_{i}^{\geq 3}\right|=d_{i}-2$, then remove from the figure the adjacent edges between vertex $v_{i}$ and the vertices of point set $V_{i}^{23}$, the new graph we derive is still a Hamilton graph.

Proof: Undirected graph $G$ is a Hamilton graph. When the vertex $v_{i}$ becomes into a second degree vertex, the variation of degree $\nabla d_{i}=d_{i}-2\left(d_{i}\right.$ is the degree of vertex $v_{i}$ in graph $\left.G\right)$. In order to ensure that the new graph is a Hamilton graph, the degree of the second degree vertex in graph $G$ does not change. If the vertex $v_{j}$ and vertex $v_{i}$ are adjacent and when vertex $v_{i}$ becomes into a second degree vertex, $\nabla d_{i} \neq 0$, then it is sure that $d_{j} \geq 3$, that is, $v_{j} \in V_{i}^{\geq 3} .\left|V_{i}^{\geq 3}\right|=d_{i}-2$ and $\nabla d_{i}=d_{i}-2$ are already known, and when vertex $v_{i}$ becomes into a second degree vertex, $\left|V_{i}^{\geq 3}\right|=0$. Assuming that after the vertex $v_{i}$ becomes into a second degree vertex, a new graph $G$ ' is obtained. if the graph $G$ 'is not a Hamilton graph, then there is no scenario where vertex $v_{i}$ becomes into a second degree vertex and the new graph does not contain a less-than-two-degree vertex, that means in the graph $G$ there does not exist a Hamilton cycle. Since this conflicts with the premise, graph $G$ ' is a Hamilton graph. Thus if we remove from the graph $G$ the adjacent edges between vertex $v_{i}$ and the vertices of point set $V_{i}^{\geq 3}$, the new graph is still a Hamilton graph.

Figure 4 shows the execution process of Theorem 1, the left side of which is a Hamilton graph containing six vertices. Choose a qualified vertex (red vertex) from the Hamilton graph and converse it to generate the Hamilton graph in the right side. 

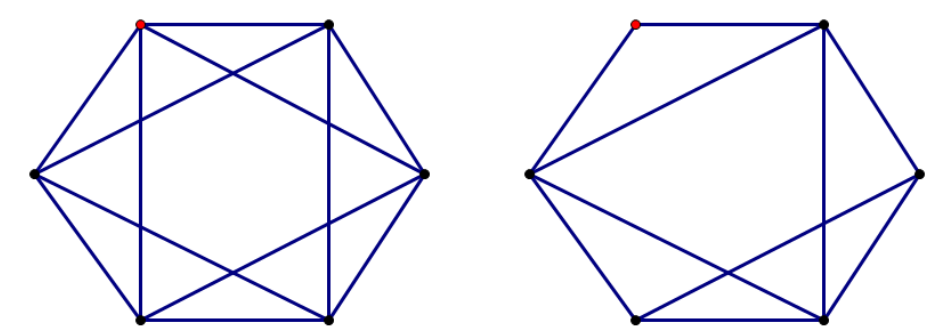

Fig. 4 Hamilton graph in the right side is conversed according to Theorem 1 into the Hamilton graph in the left portion

\section{Heuristic Search}

According to Theorem 1, it is found that vertices meeting Theorem 1's conditions can be continuously found in the undirected graph $G$ (if there is not such vertices, we could randomly select one) and transformed into a second degree vertex, this repeated process can result in a Hamilton cycle. If so, graph $G$ is a Hamilton graph. If all the scenarios are tried with an individual vertex but ending up with failure to converse it into a second degree vertex, then it shows that graph $G$ is not a Hamilton graph.

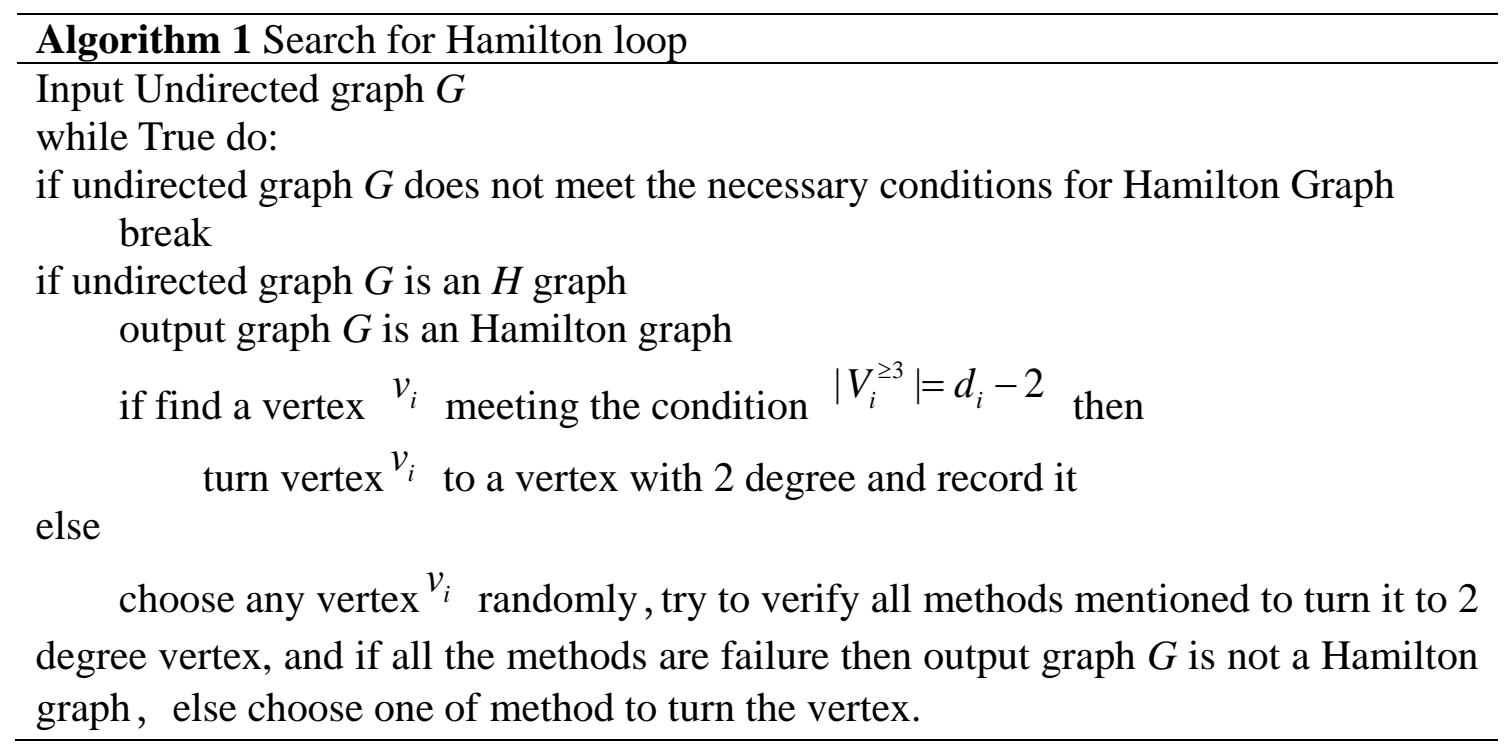

\section{The Experimental Results}

We choose the recursive search strategy as comparison strategy and the two strategies are both realized by using Python 2.7 programming. In the experiment, we use a variety of undirected Hamilton graphs and undirected non-Hamilton graphs with different sizes. The experiment was run on the PC with Inter $1.60 \mathrm{GHZ}$ and windows 7 operating system.

The results include the undirected graphs with 4 to 1000 vertices, whose relevant data are shown in Table 1 and Table 2. In the experiment we use Chinese chess as the model of the undirected graph, and use the horse-moving pattern to determine the adjacency relationship between vertices of the undirected graph. The experimental results show that the heuristic search strategy proposed in this Paper is significantly better than the recursive search strategy both in terms of computing time and the results. Time-out means that the algorithm's computing time is more than 100 seconds. Y - Hamilton graph, $\mathrm{N}$ - non-Hamilton graph. 
Table 1 Heuristic Search

\begin{tabular}{|llllllllllll|}
\hline matrix & time/s & $\mathrm{Y} / \mathrm{N}$ & matrix & time/s & $\mathrm{Y} / \mathrm{N}$ & matrix & time/s & $\mathrm{Y} / \mathrm{N}$ & matrix & time/s & $\mathrm{Y} / \mathrm{N}$ \\
\hline $2 * 2$ & 0.0049 & $\mathrm{~N}$ & $3 * 3$ & 0.0049 & $\mathrm{~N}$ & $14 * 14$ & 0.2610 & $\mathrm{Y}$ & $23 * 23$ & 2.2420 & $\mathrm{~N}$ \\
$2 * 4$ & 0.0039 & $\mathrm{~N}$ & $5 * 5$ & 0.0060 & $\mathrm{~N}$ & $15 * 15$ & 0.3459 & $\mathrm{~N}$ & $24 * 24$ & 2.7339 & $\mathrm{Y}$ \\
$4 * 4$ & 0.0039 & $\mathrm{~N}$ & $6 * 6$ & 0.0090 & $\mathrm{Y}$ & $16 * 16$ & 0.6779 & $\mathrm{Y}$ & $25 * 25$ & 3.3380 & $\mathrm{~N}$ \\
$4 * 8$ & 0.0090 & $\mathrm{Y}$ & $7 * 7$ & 0.0160 & $\mathrm{~N}$ & $17 * 17$ & 0.6960 & $\mathrm{~N}$ & $26 * 26$ & 3.7149 & $\mathrm{Y}$ \\
$8 * 8$ & 0.0239 & $\mathrm{Y}$ & $9 * 9$ & 0.0389 & $\mathrm{~N}$ & $18 * 18$ & 0.6960 & $\mathrm{Y}$ & $27 * 27$ & 4.4570 & $\mathrm{~N}$ \\
$8 * 16$ & 0.2500 & $\mathrm{Y}$ & $10 * 10$ & 0.1270 & $\mathrm{Y}$ & $19 * 19$ & 0.9470 & $\mathrm{~N}$ & $28 * 28$ & 5.3510 & $\mathrm{Y}$ \\
$16 * 16$ & 0.4580 & $\mathrm{Y}$ & $11 * 11$ & 0.1219 & $\mathrm{~N}$ & $20 * 20$ & 1.2549 & $\mathrm{Y}$ & $29 * 29$ & 5.9660 & $\mathrm{~N}$ \\
$16 * 32$ & 2.1109 & $\mathrm{Y}$ & $12 * 12$ & 0.1340 & $\mathrm{Y}$ & $21 * 21$ & 1.5030 & $\mathrm{~N}$ & $30 * 30$ & 7.0529 & $\mathrm{Y}$ \\
$32 * 32$ & 9.2750 & $\mathrm{Y}$ & $13 * 13$ & 0.2339 & $\mathrm{~N}$ & $22 * 22$ & 1.8580 & $\mathrm{Y}$ & $31 * 31$ & 8.6754 & $\mathrm{~N}$ \\
\hline
\end{tabular}

Table 2 Recursive Search

\begin{tabular}{|c|c|c|c|c|c|c|c|c|c|c|c|}
\hline matrix & time/s & $\overline{\mathrm{Y} / \mathrm{N}}$ & matrix & time/s & $\mathrm{Y} / \mathrm{N}$ & matrix & time/s & $\mathrm{Y} / \mathrm{N}$ & matrix & time/s & $\mathrm{Y} / \mathrm{N}$ \\
\hline $2 * 2$ & 0.0000 & $\mathrm{~N}$ & $3 * 3$ & 0.0049 & $\mathrm{~N}$ & $14 * 14$ & TO & - & $23 * 23$ & TO & - \\
\hline $2 * 4$ & 0.0000 & $\mathrm{~N}$ & $5 * 5$ & 10.407 & $\mathrm{~N}$ & $15 * 15$ & TO & - & $24 * 24$ & TO & - \\
\hline $4 * 4$ & 0.0160 & $\mathrm{~N}$ & $6 * 6$ & 97.584 & $\mathrm{Y}$ & $16 * 16$ & TO & - & $25 * 25$ & TO & - \\
\hline $4 * 8$ & TO & - & $7 * 7$ & TO & - & $17 * 17$ & TO & - & $26 * 26$ & TO & - \\
\hline $8 * 8$ & TO & - & $9 * 9$ & TO & - & $18 * 18$ & TO & - & $27 * 27$ & TO & - \\
\hline $8 * 16$ & TO & - & $10 * 10$ & TO & - & $19 * 19$ & TO & - & $28 * 28$ & TO & - \\
\hline $16 * 16$ & TO & - & $11 * 11$ & TO & - & $20 * 20$ & TO & - & $29 * 29$ & TO & - \\
\hline $16 * 32$ & TO & - & $12 * 12$ & TO & - & $21 * 21$ & TO & - & $30 * 30$ & TO & - \\
\hline $32 * 32$ & TO & - & $13 * 13$ & TO & - & $22 * 22$ & TO & - & $31 * 31$ & TO & - \\
\hline
\end{tabular}

\section{Conclusion}

In this paper, we propose a non-backtracking heuristic search algorithm, in which the new necessary conditions of Hamilton graph are applied flexibly to guide the search algorithm to make a faster determination of the input undirected graph. We will continue to study the relevant theory of Hamilton graph, in the hope that we can obtain a more accurate description of the algorithm to further enhance its implementation efficiency and to promote the theoretical resolution of the Hamilton graph determination.

\section{Acknowledgement}

Project for the Training of Undergraduate Students in Scientific Research granted by the Beijing Municipal Commission of Education (No.PXM2015014224000050).

\section{References:}

[1] YU Yan Kun. A Determination Method of Hamilton Graph with Matrix[J]. Journal of Educational Institute of JiLin Province, 2012,149 50

[2] Geng SuYun, QU Wan Ling, ZHANG Li Ang. Discrete Mathematics[M]. Beijing: Tsinghua University Press, 2004

[3] Vladimir G D, Bettina Klinz, et al. Exact algorithms for the Hamiltonian cycle problem in planar graphs. Operations Research Letters 2006, 34(3): 269 274

[4] Ruo W H, Maw S C. Linear time algorithms for the Hamiltonian problems on distance hereditary graphs. Theoretical Computer Science, 2005, 314(1-3): $411 \sim 440$

[5] Sohel R M, Kaykobad M. Hamiltonian cycles and Hamiltonian paths. Information Processing Letters, 2005, 94(1): $37 \sim 41$

[6] Nikolopolos S D. Parallel algorithms for Hamiltonian Problems on quasi-threshold graphs. Journal of Parallel and Distributed Computing, 2004, 64(1): $48 \sim 67$

[7] Dimakopolos V V, Palios L, et al. On the Hamiltonicity of the Cartesian product. Information Processing Letters, 2005, 31(2): $49 \sim 53$ 
[8] Amar D, Flandrin E, Gancarzewicz G. Hamiltonian cycles and paths through matchings. Electronic Notes in Discrete Mathematics, 2005, 22: $543 \sim 547$

[9] Kaneko A, Kawarabayashi K, et al. On a Hamiltonian cycle in which specified vertices are not isolated. Discrete Mathematics, 2002, 258(1-3): 85 91

[10] Frieze A, Krivelevich M. On Packing Hamilton cycle in $\varepsilon$-regular graphs. Journal of Combinatorial Theory, Series B, 2005, 94(1): $159 \sim 172$

[11] Li X W, Wei B, Yu Z G, et al. Hamilton cycles in 1-tough triangle free graphs. Discrete Mathematics, 2002, 254(1-3): $275 \sim 287$

[12] Li G J, Lu M, Liu Z. Hamiltonian cycles in 3-connected Claw-free graphs. Discrete Mathematics, 2002, 250(1-3): $137 \sim 151$

[13] Kawarabayashi K, Ota K, Saito A. Hamiltonian cycles in n-factor critical graphs. Discrete Mathematics, 2001, 240(1-3): $71 \sim 82$

[14] Tambouratzis T. Solving the Hamiltonian cycle problem via an artificial neural network. Information Processing Letters, 2000, 75(6): $273 \sim 242$ 